\title{
Prejuicios y estereotipos negativos que perciben los niños de emigrantes de sus compañeros de escuela
}

\author{
Villavicencio Alvarado F., Orellana Méndez A., Tenorio Ambrosi P. \\ Proyecto VLIR: Migración Internacional y Desarrollo Local, Facultad de Psicología, Universidad de \\ Cuenca, Cuenca, Ecuador
}

Autor para correspondencia: fervillavicencio1@ @otmail.com

Fecha de recepción: 11 de enero 2012 - Fecha de aceptación: 13 de mayo 2012

\begin{abstract}
RESUMEN
Existe un discurso social cargado de atributos negativos hacia los niños de los emigrantes y que puede evidenciarse en el contexto educativo; por ello, el objetivo de la investigación es identificar los prejuicios y estereotipos que perciben los niños de emigrantes y que provienen de sus compañeros-as de escuela que no son niños de emigrantes. Participaron 384 niños de migrantes de cuarto a séptimo año de Educación Básica de 18 escuelas públicas y privadas de los cantones Cuenca, Girón y Gualaceo pertenecientes a la Provincia del Azuay, Ecuador. Las variables de la investigación se midieron a través de un cuestionario compuesto por dos escalas: la primera, la escala de prejuicio sutil y manifiesto de Pettigrew y Meertens (1995) adaptada por Rueda y Navas (1996), y la segunda, elaborada por los autores de esta investigación. La investigación concluye que no existen manifestaciones evidentes de prejuicio sutil y manifiesto, desde la percepción de los niños de emigrantes. En cuanto a los estereotipos, se deduce que estos niños sí perciben que sus compañeros-as los estereotipan de forma negativa como: ociosos, groseros, interesados en el dinero, que utilizan ropa fea y que proceden de una clase social inferior. Además, sienten que sus compañeros-as consideran que no son limpios y ordenados, que no juegan y comparten con ellos, que no les quieren, que no son personas simpáticas, que no tienen familia y que no tienen quien les defienda.
\end{abstract}

Palabras clave: Emigración, niños, prejuicio, discriminación, estereotipos, escuela.

\begin{abstract}
A social discourse fed by prejudiced attitudes and ideologies exists towards children of migrants with the particular risk of social exclusion in the educational context; a topic of debate and concern for the society. The research presented in this paper aims at identifying prejudices and stereotypes perceived by emigrant children created by classmates children of no emigrant families. A total of 384 children of emigrant families belonging to the fourth through the seventh year of basic education from 18 public and private schools in the cantons Cuenca, Girón and Gualaceo belonging to the Province of Azuay, Ecuador, participated in the research. The research variables were measured through a questionnaire by means of two scales: the first one the subtle and blatant prejudice scale developed by Pettigrew and Meertens (1995) and further adapted by Rueda and Navas (1996), and a second one developed by the authors. The research concludes that there are no obvious signs of manifest and subtle prejudice, based on the perception of the children belonging to migrant families. In terms of stereotypes, those children perceive that their classmates negatively stereotype them as lazy, rude, interested in money, using ugly clothes and coming from a lower social class. In addition, they feel that their classmates consider them as no clean and tidy, that they are less inclined to play and share their time with them, not loving them, treated as not nice people, having no family, not being defended.
\end{abstract}

Keywords: Migration, children, prejudice, discrimination, stereotypes, school. 


\section{INTRODUCCIÓN}

En el discurso social ecuatoriano que se reproduce en diferentes medios de comunicación como diarios, telediarios, etc. ${ }^{1}$ sobre el emigrante y su familia se le asigna ciertos atributos y comportamientos negativos, por ejemplo se dice que los niños de emigrantes son "malos estudiantes" y que "son un problema en la escuela por su conducta agresiva". En este contexto existe la posibilidad de que esta población pueda ser afectada por actitudes de rechazo por parte de sus compañeros que no son niños de emigrantes, puesto que esta caracterización negativa es trasmitida a los niños y éstos, a su vez, los reproducen en la escuela, asignando características negativas a los niños de emigrantes, y que son percibidas por éstos. Al respecto, entre otros estudios, la investigación de Benedictis y Calfat (2010), realizada en el cantón San Fernando (Provincia del Azuay, Ecuador), puso de manifiesto que las calificaciones en rendimiento escolar y conducta son iguales entre los niños de emigrantes y los que no tienen progenitores migrantes. Datos que contradicen al discurso social sobre la familia emigrante.

Una breve contextualización de la emigración en el Ecuador nos permite ubicarla desde hace aproximadamente 60 años y está caracterizada por dos diásporas plenamente identificadas. La primera, de los años 50 del siglo pasado, denominada "crisis de la paja toquilla" y cuyo destino principal de emigración fue Estados Unidos de América. La segunda, a finales de los años 90, marcada por la "crisis bancaria" y cuyo destino fue Europa y Estados Unidos de América. Según el Instituto Nacional Ecuatoriano de Estadísticas y Censos (INEC, 2008) utilizando varias fuentes, señala que 1,57 millón de ecuatorianos emigraron a otros países. Los principales países de destino son: España, en donde se concentra el 48,6\%, seguido de los Estados Unidos con el 28,2\% e Italia con el 10,8\%; se trata mayoritariamente de la emigración registrada legalmente. En la última encuesta del Ecuador sobre migración (INEC, 2008) el 31\% de migrantes ecuatorianos habían dejado niños menores de 18 años en el país. El 64,4\% de los migrantes son jóvenes entre 19 y 29 años, edades en las que se incorporan a la vida productiva. El 7,7\% son menores de 15 años, el 4,9\% son mayores de 30 años.

El VII Censo de Población y VI de Vivienda (INEC, 2010) señala que el 7,34\% del total de hogares del Ecuador tienen al menos un miembro que ha emigrado al exterior. En la Provincia del Azuay, la cifra de hogares censados asciende a 188.765; de los cuales, 27.503 declaran que al menos uno de sus miembros salió de la provincia en el último período intercensal (2001-2010), equivalente al 14,57\% del total de hogares censados. En Cuenca son 17.062 hogares con miembros migrantes $(12,7 \%)$, en Girón $724(1,8 \%)$ y en Gualaceo $2.647(5,8 \%)$ del total de hogares que tienen al menos un miembro en el exterior en cada uno de los tres cantones de estudio. La edad promedio de los que emigraron en Cuenca es de 27,53 años, Girón 26,74 años y Gualaceo de 25,03 años. En cuanto al sexo de los emigrantes, en Cuenca el 64,4\% fueron hombres, en Girón el 67,5\% y en Gualaceo el 68,8\%. Según Ortiz y col. (2007) que realizó un censo en la Provincia del Azuay de niños de emigrantes en el sistema educativo, señala que en Girón se matricularon 1.937 niños en las 27 escuelas, de los cuales el $31,6 \%$ eran niños de padres emigrantes; en Gualaceo se matricularon 8.254 niños en 62 escuelas, el $32,6 \%$ eran niños de padres emigrantes; y en Cuenca, se tomó una muestra de 74 escuelas que tenían matriculados a 17.477 niños, de los cuales $16,9 \%$ eran niños de padres emigrantes. En total son 6.269 niños y niñas escolarizados que tienen a uno o ambos padres emigrantes en tan solo tres cantones de la Provincia del Azuay; lo que deja ver la magnitud de niños escolarizados que son hijos de emigrantes.

Es precisamente en el sistema educativo donde se desarrollan los procesos de socialización más concretos y complejos en los niños; así, Casal (2006) afirma que "los intercambios interpersonales

\footnotetext{
${ }^{1}$ Cuando un familiar migra a otro país, se produce una ruptura de los lazos emocionales de cada uno de los miembros. En el caso de los niños, su comportamiento y sus valores cambian y su disciplina de carácter conductual queda fragmentada. "Puede tomar diferentes actitudes, pero muchas veces se relacionan con la rebeldía, no quieren hacer deberes, son indisciplinados, no se quieren asear o son desordenados" (www.eltelegrafo.com.ec; consultado el 8 de octubre del año 2008).
} 
que se generan en el aula van a condicionar el contexto y el clima social en el que se desarrolla el proceso de enseñanza-aprendizaje de los niños". García y col. (2003) exponen que los niños, en su proceso de socialización, tienen la capacidad desde edades muy tempranas, de categorizar a las personas en función de diversos grupos sociales, facilitando la adquisición de estereotipos sobre algunos grupos y pudiendo utilizarlos en sus relaciones con los miembros de estos grupos. La teoría de la Identidad Social (Tajfel, 1970, citado en Tajfel y Turner, 1979) confirma la idea de que los niños, desde edades muy tempranas, son capaces de distinguir entre exogrupos con características diferenciales y sus percepciones dependen, igual que las de los adultos, de las distintas posiciones sociales que ocupan dichos grupos.

Si bien, en nuestro sistema escolar, no se habla abiertamente de prejuicios a niños de migrantes o a otros grupos (p.e. negros, indígenas, niños de inmigrantes colombianos o peruanos), no quiere decir que éstos no existan de manera expresa o sutil; de ahí, el objetivo principal de este trabajo que es el de identificar la presencia del prejuicio expresada en uno de sus componentes que es el estereotipo, en este caso dirigido a los niños de emigrantes. Allport (1962) en La Naturaleza del Prejuicio afirma que "el prejuicio es una actitud hostil o prevenida hacia una persona que pertenece a un grupo, simplemente porque pertenece a ese grupo, suponiéndose por lo tanto que posee las cualidades objetables atribuidas al grupo". Myers (2000) define el prejuicio como "una pre concepción negativa hacia un grupo y hacia sus miembros individuales, es una actitud negativa que nos sesga contra una persona basándose únicamente en la identificación con un grupo en particular". Para verificar la presencia de prejuicios entre niños de emigrantes en el ámbito escolar, este estudio utilizó la escala adaptada por Rueda y Navas (1996) de la escala original de Pettigrew y Meertens (1995), que está formada por dos subescalas. La primera llamada prejuicio manifiesto, constituida por los siguientes componentes: percepción de amenaza y rechazo del exogrupo y oposición al contacto íntimo con los miembros del exogrupo; la segunda denominada prejuicio sutil y sus componentes son: defensa de los valores tradicionales, exageración de las diferencias culturales y negación de emociones positivas hacia el exogrupo. Pettigrew y Meertens (1995) realizan una distinción entre dos tipos de prejuicio: el manifiesto y el sutil. El primero corresponde al prejuicio antiguo o tradicional, y se caracteriza por el rechazo abierto hacia el exogrupo (p.e. solo el hecho de ser niños de emigrantes) y la percepción de amenaza al endogrupo (p.e. económico o social), también se expresa con rechazo al contacto de las relaciones cercanas con el exogrupo. El principal aporte de estos autores es la concepción del prejuicio sutil, que se basa en la defensa de los valores tradicionales y esto se expresa en conductas encubiertas, a diferencia del manifiesto cuyas conductas son abiertas y notorias. En Introducción a la Psicología Social de Gaviria y col. (2009) acerca de la estructura de las actitudes, Devine (1995), citado en Fiske (2004), señalan que "el prejuicio, como cualquier actitud, estaría formado por tres componentes: un componente afectivo -sentimientos y emociones- que suscitan las personas a las que se dirige el prejuicio, un componente cognitivo -denominado estereotipo- y un componente conativo o conductual -conocido como discriminación-".

Por lo tanto, los estereotipos son un conjunto de creencias compartidas sobre las características de un grupo social, en nuestro caso los niños de migrantes; éstas constituyen un tipo específico de esquema cognitivo de gran fuerza sobre la información social que recibimos. Queda definido entonces que, el componente cognitivo del prejuicio lo constituyen los estereotipos, como lo dice Fiske (2004) y Gaviria y col. (2009). Éste componente está dado por la información y el conocimiento que las personas adquieren o creen que tienen sobre los objetos actitudinales; en otras palabras, por las creencias que ellos tienen respecto a otro grupo social; pudiendo ser éstas positivas o negativas, es decir favorables o desfavorables hacia el objeto; se trata pues, de un conjunto de atributos adquiridos socialmente, que tienen o utilizan los miembros de un grupo (endogrupo) para definir o caracterizar a los miembros de otro grupo (exogrupo) y que necesariamente son expresadas en el discurso. Brigham (1971) y Montes Berges (2008) señala que los estereotipos son "generalizaciones hechas sobre un grupo, concernientes a la atribución de rasgos, que es considerada como injustificada por un observador". Así, el expresar que todos los niños de emigrantes, en el ámbito escolar, son malos estudiantes, se está etiquetando a todos los niños dentro de la categoría "malos estudiantes". Gaviria y col. (2009) explican que la categorización genera efectos automáticos, siendo uno de ellos la homogeneidad exogrupal, que consiste en percibir a los miembros del otro grupo más semejantes entre sí y a los miembros del grupo al que pertenecemos como más diferenciados. 
De lo anterior, se desprende el objetivo fundamental de la investigación, que es identificar los prejuicios y estereotipos que perciben los niños de emigrantes y que provienen de sus compañeros de escuela que no son niños de emigrantes.

\section{MATERIALES Y MÉTODOS}

La muestra está compuesta por 384 niños matriculados de cuarto a séptimo año de educación básica durante el periodo escolar 2010-2011 de los cantones Cuenca, Girón y Gualaceo pertenecientes a la provincia del Azuay en Ecuador, de los cuales el 48,7\% son mujeres y 51,3\% varones; las edades oscilan entre los 8 y 12 años, con una edad media de los niños de 10 años y la desviación típica de 1,10. Se clasificó a las escuelas de acuerdo al porcentaje de niños de emigrantes, en tres grupos excluyentes: (1) escuelas con alto porcentaje de niños de emigrantes (65\% o más), (2) escuelas con porcentaje medio entre niños de emigrantes e niños de no emigrantes (entre el 40 y 65\%), y (3) escuelas con baja proporción de niños de emigrantes (menor de 40\%).

Las variables de la investigación se midieron a través de un cuestionario compuesto por dos escalas. La primera para medir estereotipos y la segunda para medir prejuicio sutil y manifiesto. Previo a la aplicación de los cuestionarios, se realizó la fase de pilotaje, que permitió reducir el número de ítems de 150 a 26. La primera escala de estereotipos fue elaborada por el equipo investigador. La etapa de pilotaje estaba constituida por una serie de 48 enunciados positivos y negativos que después se redujeron a 11 ítems definitivos distribuidos en: 6 ítems para los estereotipos positivos: son limpios y ordenados, juegan y comparten con todos, les quieren a ustedes, son chéveres $^{2}$ personas, tienen familia, tienen quien les defiendan; y 5 ítems para estereotipos negativos: ociosos, groseros, interesados en el dinero, utilizan ropa fea y parecen cholos ${ }^{3}$.

La segunda escala utilizada fue la adaptación de Rueda y Navas (1996) que hacen de la escala original de Prejuicio Sutil y Manifiesto de Pettigrew y Meertens (1995), en esta investigación las subescalas quedaron constituidas de la siguiente manera: la primera, del prejuicio manifiesto con los componentes: percepción de amenaza y rechazo del exogrupo con 8 ítems, y evitación al contacto íntimo con los miembros del exogrupo con 3 ítems; y la segunda subescala del prejuicio sutil con el componente negación de habilidades y capacidades con 4 ítems. A cada una de las opciones de respuesta se les asignó una valoración; en el caso de los ítems positivos las valoraciones otorgan un rango de 1 Siempre a 4 Nunca; y en el caso de los negativos la valoración 4 Siempre a 1 Nunca. El criterio de medición para interpretar la escala es: a más alto puntaje existe mayor evidencia de prejuicio. Los cuestionarios se administraron de manera colectiva, reuniendo a los niños de emigrantes en sendas sesiones de aproximadamente 20 minutos para cada escuela. Una vez entregado los cuestionarios, y antes de iniciar con el desarrollo, se procedió a ejemplificar cómo responder a cada ítem.

Para verificar la validez de los constructos se aplica la técnica del análisis factorial, utilizando el método de componentes principales con rotación Varimax. Esta técnica es apropiada en este caso, ya que permite transformar un número de variables originales a otro conjunto de variables llamadas componentes principales, que son combinaciones lineales de las primeras. La utilidad de esta técnica reside en que con ella se puede reducir un número grande de variables correlacionadas entre sí a un número manejable de variables no relacionadas y que explican una proporción de la varianza correspondiente a las variables originales. En otras palabras, la idea es seleccionar un número de componentes menor al número de variables originales con la ventaja de que estos incorporan gran parte de la información original. Una importante característica de los componentes es que éstos suelen correlacionarse altamente con un subconjunto de variables originales al mismo tiempo que pueden tener baja correlación con otro subconjunto, dando lugar a una nueva denominación de variables.

\footnotetext{
${ }^{2}$ Chéveres: agradable, buena persona y con quien es bueno tratar

${ }^{3}$ Cholos: término periorativo para decir que la persona es indio o de origen campesino
} 
El análisis factorial puede conllevar a una indeterminación debida a que cualquier base de la matriz de solución es también una solución, por lo que intuitivamente será más fácil interpretar un componente cuando éste se encuentre más cerca de las variables observadas, lo que puede lograrse rotando los factores. En palabras de Uriel y Aldás (2005) "Cuando se aplica el análisis factorial se trata de que los factores comunes tengan una interpretación clara, porque de esa forma se analizan mejor las interrelaciones existentes entre las variables originales. Sin embargo, en muy pocas ocasiones resulta fácil encontrar una interpretación adecuada de los factores iniciales, con independencia del método que se haya utilizado para su extracción. Precisamente los procedimientos de rotación de factores se han ideado para obtener, a partir de la solución inicial, unos factores que sean fácilmente interpretables".

Para la validación del ajuste general de los componentes se utilizó el coeficiente alfa de Cronbach (1951). Es un índice usado para medir la confiabilidad del tipo consistencia interna de una escala, es decir, para evaluar la magnitud en que los ítems de un instrumento están correlacionados. En otras palabras, el alfa de Cronbach es el promedio de las correlaciones entre los ítems que hacen parte de un instrumento. También se puede concebir este coeficiente como la medida en la cual algún constructo, concepto o factor medido está presente en cada ítem. Generalmente, un grupo de ítems que explora un factor común muestra un elevado valor de alfa de Cronbach. El coeficiente alfa de Cronbach permite cuantificar el nivel o grado de fiabilidad de una escala de medida para representar una magnitud no observable. Dicho coeficiente está definido para el rango entre 0 y $1^{4}$. Si los ítems son independientes entre sí (es decir no constituyen conjuntamente una escala fiable) el valor del coeficiente alfa será 0 . Por el contrario, para ítems muy correlacionados entre sí, el coeficiente alfa estará muy cercano a la unidad. Empíricamente se considera que un coeficiente alfa empieza a ser aceptable a partir de puntuaciones por sobre 0,65 .

Para validar si existen diferencias significativas en el promedio de los estereotipos de acuerdo al género, al tipo de escuela (particular y pública) y a la ubicación de la escuela (urbana y rural), se procedió a aplicar un test de diferencia de medias para determinar si existen diferencias estadísticamente significativas entre los diversos grupos considerados en este estudio dentro de las variables categóricas 5 .

Prueba t para la igualdad entre medias.

\begin{tabular}{lcccc}
\hline \multirow{2}{*}{ Variable categórica } & \multicolumn{2}{c}{ Estereotipo positivo } & \multicolumn{2}{c}{ Estereotipo negativo } \\
\cline { 2 - 5 } & \multicolumn{1}{c}{ Valor $\mathrm{t}$} & Valor $\mathrm{p}$ & Valor $\mathrm{t}$ & Valor $\mathrm{p}$ \\
\hline Sexo & $(-4,366)$ & $0,00^{* * *}$ & 1,957 & $0,051^{*}$ \\
Tipo de escuela & 1,144 & 0,253 & 1,134 & 0,258 \\
Ubicación & $(-2,066)$ & $0,039^{* *}$ & $(-1,474)$ & 0,141 \\
\hline
\end{tabular}

* Significativa al 10\%, ** Significativa al 5\%; *** Significativa al $1 \%$

\footnotetext{
${ }^{4}$ El coeficiente alfa de Cronbach se calcula utilizando la siguiente expresión $\alpha=\left[\frac{k}{k-1}\right]\left[1-\frac{\sum_{i=1}^{k} S_{i}^{2}}{S_{t}^{2}}\right]$, donde $S_{i}^{2}$ es la varianza del ítem $i . S_{t}^{2}$ es la varianza de la suma de todos los ítems correspondientes al constructo específico. Finalmente $k$ representa el número de ítems o preguntas de cada constructo.

${ }^{5}$ Se contrastaron las hipótesis nulas de que no existen diferencias significativas entre las medias obtenidas para los subgrupos: sexo (masculino, femenino), tipo de escuela (fiscal, particular) y ubicación (urbana, rural), contra las hipótesis alternativas de que efectivamente hay diferencias reales no explicadas por error de muestreo en dichos subgrupos.
} 


\section{RESULTADOS}

\subsection{Descripción de los ítems de las escalas}

El cuestionario con las dos escalas está estructurado de la siguiente manera: la escala de prejuicio está identificada por los ítems del 12 al 26 de la escala adaptada de Pettigrew y Meertens (1995) descrita en el epígrafe anterior, en tanto que los ítems del 1 al 11 describen a los estereotipos, utilizando la escala propuesta en el presente estudio. En las Tablas 1 y 2 se presentan los promedios y las desviaciones estándar (DS) de cada uno de los ítems considerados.

Para analizar adecuadamente los resultados presentados en la Tabla 1, es importante separar los ítems negativos de los positivos. Se observa claramente que de acuerdo a su promedio, ninguno de los ítems negativos de la escala de Pettigrew y Meertens (1995) (ítems 12 al 26) supera en promedio el valor de 2. De esta forma, el ítem negativo «Podrían ser buenos alumnos si se dedicaran más al estudio» representa el atributo con mayor carga de prejuicio percibido $(1,95)$, mientras que el ítem «Las personas que les cuidan a ustedes les enseñan malas costumbres» es el atributo que muestra un menor prejuicio percibido $(1,22)$. Si consideramos los ítems positivos, el ítem «Sienten admiración por ustedes» presenta el promedio con mayor evidencia de prejuicio $(2,47)$, y el promedio con menor carga de prejuicio $(1,43)$ está representado por el ítem «Sí les gusta tenerles a ustedes como compañeros». En términos generales, estos valores permiten concluir que no existen manifestaciones evidentes de prejuicio desde la percepción de los niños de emigrantes, de acuerdo a la escala de Pettigrew y Meertens (1995).

Tabla 1. Estadísticos descriptivos, escala de prejuicio. ${ }^{6}$

\begin{tabular}{rlcc}
\hline$\#$ & Ítem & M & DS \\
\hline 12 & Si les gusta tenerles a ustedes como compañeros & 1,43 & 0,82 \\
\hline 13 & Si se puede confiar en ustedes & 1,91 & 1,06 \\
\hline 14 & Pueden ser buenos deportistas a pesar de no tener a sus papás cerca & 2,02 & 1,20 \\
\hline 15 & Hacen quedar mal a la escuela & 1,35 & 0,81 \\
\hline 16 & Son inteligentes y por eso sacan buenas notas & 2,21 & 1,07 \\
\hline 17 & Podrían ser buenos alumnos si se dedicaran mas al estudio & 1,95 & 1,17 \\
\hline 18 & No hacen tareas con ustedes porque ustedes les caen mal & 1,52 & 0,97 \\
\hline 19 & Los papás de sus amigos no les dejan ir a jugar en su casa & 1,81 & 1,13 \\
\hline 20 & Los papás de sus amigos si les dan permiso para jugar con ustedes & 2,02 & 1,14 \\
\hline 21 & Deberían estar en otra aula & 1,35 & 0,80 \\
\hline 22 & No deberían estar en esta escuela & 1,40 & 0,87 \\
\hline 23 & Las personas que les cuidan a ustedes les enseñan malas costumbres & 1,22 & 0,63 \\
\hline 24 & Ustedes no respetan las normas de la escuela & 1,58 & 1,03 \\
\hline 25 & Sienten admiración por ustedes & 2,47 & 1,18 \\
\hline 26 & Se llevan con ustedes solo por la plata & 1,38 & 0,84 \\
\hline
\end{tabular}

En cuanto a los ítems del 1 al 11 en la Tabla 2, los promedios obtenidos indican que los niños de emigrantes perciben en mayor o menor grado la presencia de estereotipos por parte de sus pares de acuerdo a la escala utilizada. Encontramos que «Groseros-agresivos» es el ítem negativo con menor promedio $(3,54)$ indicando menor intensidad de estereotipo, mientras que «Parecen cholos» es el ítem negativo que muestra mayor intensidad de estereotipo, con un promedio de 3,74. Para los ítems positivos «Son limpios y ordenados» muestra un menor intensidad de estereotipo con un promedio de

\footnotetext{
${ }^{6}$ Numero de encuestas es $384, \mathrm{M}=$ promedia, DS = desviación estándar
} 
2,77, mientras que en el ítem «Tus compañeros que no son niños de migrantes les quieren a ustedes» expresa el mayor intensidad de estereotipo por parte del endogrupo, con una media de 3,14.

Una primera aproximación a la explicación de estos resultados es que los niños escuchan en el discurso de sus compañeros atributos negativos expresados hacia ellos por ser niños de emigrantes; estos atributos negativos son identificados en el estudio como estereotipos, los cuales, individualmente o en conjunto, marcan características en ellos como pertenecientes a un exogrupo: ser niños de emigrantes. Tal es el caso, por ejemplo, del ítem 1 «Tus compañeros, que no son niños de emigrantes, dicen que ustedes son groseros-agresivos en la escuela». Aquí el atributo negativo es grosero-agresivo, y la media estadística de este ítem alcanza un valor de 3,54 que lo ubica en la categoría de Siempre; es decir, los niños conocen que a ellos se les atribuye la característica de ser groseros-agresivos por ser niños de emigrantes. En definitiva, no hay evidencia de que los niños de emigrantes perciben prejuicio sutil y manifiesto por parte de sus pares, de acuerdo a la escala de Pettigrew y Meertens (1995). Sin embargo, en cuanto a los estereotipos -cuya escala es propuesta para el presente estudio- la situación es distinta: los niños perciben que sus compañeros les asignan atributos negativos por ser niños de emigrantes.

Tabla 2. Estadísticos descriptivos estereotipos. ${ }^{7}$

\begin{tabular}{clcc}
\hline$\#$ & Ítem & $\mathrm{M}$ & $\mathrm{DS}$ \\
\hline 1 & Ociosos & 3,57 & 0,84 \\
\hline 2 & Groseros & 3,54 & 0,80 \\
\hline 3 & Interesados en el dinero & 3,66 & 0,81 \\
\hline 4 & Son limpios y ordenados & 2,77 & 1,25 \\
\hline 5 & Utilizan ropa fea & 3,70 & 0,72 \\
\hline 6 & Juegan y comparten con todos & 3,04 & 1,12 \\
\hline 7 & Parecen cholos & 3,74 & 0,64 \\
\hline 8 & Les quieren a ustedes & 3,14 & 1,05 \\
\hline 9 & Son chéveres personas & 2,92 & 1,14 \\
\hline 10 & Si tienen familia & 3,12 & 1,20 \\
\hline 11 & Si tienen quien les defienda & 3,01 & 1,20 \\
\hline & & & \\
\hline
\end{tabular}

En el análisis factorial se encontró que en la escala de prejuicio sutil y manifiesto los ítems se agruparon en tres componentes. Al primero se lo denominó componente Amenaza-Rechazo y está compuesto por 8 ítems. Este mide el rechazo y la percepción de amenaza por parte del endogrupo (niños de no emigrantes) hacia el exogrupo (niños de emigrantes); al segundo componente se lo etiquetó como Negación al Contacto Íntimo e incluye 3 ítems; este componente mide el rechazo al contacto íntimo y las relaciones cercanas con el exogrupo. Finalmente, al tercer componente que agrupa cuatro ítems, se lo denominó Negación de las Capacidades y Habilidades; en este caso el endogrupo considera que el exogrupo no tiene destrezas físicas, intelectuales y sociales para destacarse en relación con sus otros pares. Estos tres componentes explican en conjunto el 45,5\% de la varianza: $19,9 \%$ el primer componente, $14 \%$ el segundo y $11,6 \%$ el tercer componente.

La Tabla 3 es la matriz factorial obtenida utilizando los ítems 12 al 26. Se han reproducido únicamente las saturaciones correspondientes a tres factores con valores propios mayores a uno. Aplicando una lectura más minuciosa a los resultados expresados en esta tabla, observamos que el ajuste general de los ítems dentro de cada componente no es del todo satisfactorio. Específicamente, gran parte de las correlaciones estimadas correspondientes a los componentes 1 y 3 son muy bajas.

\footnotetext{
${ }^{7}$ Numero de encuestas es $384, \mathrm{M}=$ promedia, DS = desviación estándar
} 
Tabla 3. Componentes del prejuicio con tres variables reagrupadas.

\begin{tabular}{|c|c|c|c|c|}
\hline \multirow[b]{2}{*}{$\#$} & \multirow[b]{2}{*}{ Ítems } & \multicolumn{3}{|c|}{ Variables (componentes) } \\
\hline & & $\begin{array}{l}\text { Rechazo- } \\
\text { amenaza }\end{array}$ & $\begin{array}{l}\text { Evitación al } \\
\text { contacto }\end{array}$ & $\begin{array}{r}\text { Negación de } \\
\text { habilidades }\end{array}$ \\
\hline 12 & Si les gusta tenerles como compañeros & & 0,725 & \\
\hline 13 & Si se puede confiar en ustedes & & 0,628 & \\
\hline 14 & $\begin{array}{l}\text { Pueden ser buenos deportistas a pesar de no } \\
\text { tener a sus papás cerca }\end{array}$ & & & 0,423 \\
\hline 15 & Hacen quedar mal a la escuela & 0,511 & & \\
\hline 16 & Son inteligentes y por eso sacan buenas notas & & & 0,730 \\
\hline 17 & $\begin{array}{l}\text { Podrían ser buenos alumnos si se dedicaran más } \\
\text { al estudio }\end{array}$ & & & 0,442 \\
\hline 18 & $\begin{array}{l}\text { No hacen tareas con ustedes, porque ustedes les } \\
\text { caen mal }\end{array}$ & 0,567 & & \\
\hline 19 & $\begin{array}{l}\text { Los papás de sus amigos no les dejan ir a jugar } \\
\text { en su casa }\end{array}$ & 0,362 & & \\
\hline 20 & $\begin{array}{l}\text { Los papás de sus amigos si les dan permiso para } \\
\text { jugar con ustedes }\end{array}$ & & 0,666 & \\
\hline 21 & Deberían estar en otra aula & 0,717 & & \\
\hline 22 & No deberían estar en esta escuela & 0,721 & & \\
\hline 23 & $\begin{array}{l}\text { Las personas que les cuidan a ustedes les } \\
\text { enseñan malas costumbres }\end{array}$ & 0,685 & & \\
\hline 24 & Ustedes no respetan las normas de la escuela & 0,584 & & \\
\hline 25 & Sienten admiración por ustedes & & & 0,633 \\
\hline 26 & Se llevan con ustedes solo por la plata & 0,585 & & \\
\hline
\end{tabular}

En relación a los ítems del 1 al 11, el análisis factorial confirmó la existencia de dos componentes de los estereotipos, que se manifiestan como estereotipos positivos y negativos. El componente Estereotipos positivos incluye a los ítems: son limpios y ordenados, juegan y comparten con todos, les quieren a ustedes, son chéveres personas, tienen familia, tienen quien les defiendan. En el componente Estereotipos negativos, confluyen los ítems: son ociosos, son groseros, son interesados en la plata, utilizan ropa fea, parecen cholos. La matriz factorial obtenida se reproduce en la Tabla 4. Nuevamente, en dicha tabla solo se han reproducido las saturaciones a dos factores con autovalores mayor a la unidad. Los resultados muestran altas correlaciones entre los ítems y sus respectivos componentes, de manera que se puede afirmar que las escalas aplicadas utilizando los ítems 1 al 11 resultan confiables para medir los estereotipos.

\subsection{Valoración del ajuste general de los factores}

Con respecto a los estereotipos los resultados indican un coeficiente $\alpha$ de 0,77 para el componente Estereotipos Positivos, mientras que para el componente Estereotipos Negativos se obtuvo un coeficiente $\alpha$ de 0,67 . En la escala de prejuicio manifiesto y sutil los resultados indican que el componente Amenaza-Rechazo presenta un coeficiente $\alpha$ de 0,72, el componente Negación al Contacto Íntimo registró un valor del coeficiente $\alpha$ de 0,57 y finalmente el coeficiente $\alpha$ para Negación de las Capacidades y Habilidades fue de 0,66. En definitiva, basados en el coeficiente $\alpha$ de Cronbach, se puede afirmar que el nivel de fiabilidad de las escalas de medida para los componentes asociados a los estereotipos son valores aceptables, mientras que la fiabilidad para los componentes asociados a los prejuicios manifiesto y sutil no es del todo adecuada. 
Tabla 4. Matriz de componentes rotados. ${ }^{8}$

\begin{tabular}{llcc}
\hline \multirow{2}{*}{ Ítems } & \multicolumn{2}{c}{ Variables (componentes) } \\
\cline { 2 - 4 } & & Estereotipos positivos & Estereotipos negativos \\
\hline 1 & Ociosos & & 0,687 \\
\hline 2 & Groseros & & 0,611 \\
\hline 3 & Interesados en el dinero & 0,617 & \\
\hline 4 & Son limpios y ordenados & & 0,640 \\
\hline 5 & Utilizan ropa fea & 0,701 & \\
\hline 6 & Juegan y comparten con todos & & \\
\hline 7 & Parecen cholos & 0,717 & \\
\hline 8 & Les quieren a ustedes & 0,713 & \\
\hline 9 & Son chéveres personas & 0,657 & \\
\hline 10 & Si tienen familia & 0,704 & \\
\hline 11 & Si tienen quien les defiendan & & \\
\hline
\end{tabular}

\subsection{Comparación de las variables latentes de acuerdo al género de los estudiantes, ubicación y tipo de escuela}

Debido a que no se encontró evidencia suficiente que valide los componentes del prejuicio sutil y manifiesto, el análisis que prosigue solamente se enmarcará en el estudio de los estereotipos. De esta forma, a partir de los resultados obtenidos en el análisis factorial se procedió a construir las variables latentes para la escala de estereotipos. La puntuación asignada a cada componente no es más que el promedio de los ítems que correlacionan fuertemente con éste. La Tabla 5 resume las medias y las desviaciones típicas de cada uno de los componentes: Estereotipo Positivo y Estereotipo Negativo. Los resultados indican que hay una fuerte percepción de estereotipo negativo, con un promedio de 3,64 , mientras que la percepción del estereotipo positivo, si bien es menor al considerar las variables re-escaladas, muestra un promedio de 3,0.

Tabla 5. Estereotipos positivos y negativos

\begin{tabular}{clcc}
\hline$\#$ & Ítem & $\mathrm{M}$ & $\mathrm{DS}$ \\
\hline 1 & Estereotipo positivo & 3,00 & 0,80 \\
\hline 2 & Estereotipo negativo & 3,64 & 0,50 \\
\hline
\end{tabular}

De acuerdo a la Tabla 5, no se pudo rechazar la hipótesis de que existen diferencias significativas en la valoración promedio del componente de los estereotipos positivos de acuerdo al género ( $\mathrm{p}=$ $0,00)$ y a la ubicación $(\mathrm{p}=0,039)$. En cuanto al componente de los estereotipos negativos en promedio estos se diferencian únicamente por el género de los niños, pero solo si consideramos un nivel de significancia del $10 \%(\mathrm{p}=0,051)$.

En definitiva, la evidencia indica que son las niñas el grupo más vulnerable a los atributos negativos; dado que ellas perciben más que sus compañeros varones estereotipos negativos y además perciben en promedio menos estereotipos positivos que sus pares masculinos.

\footnotetext{
${ }^{8}$ La matriz de componentes rotada es una nueva matriz que tiene una interpretación más clara. Conociendo qué significan las variables que están altamente correlacionadas con cada uno de los componentes se puede ofrecer una interpretación de estos asociada a la variable adecuación del centro de impactos.

Método de extracción: Componentes principales.

Método de rotación: Normalización Varimax.
} 


\section{DISCUSIÓN}

Conforme a la escala de prejuicio sutil y manifiesto de Pettigrew y Meertens (1995), adaptada por Rueda y Navas (1996), no se identificó la presencia de prejuicio. Al respecto podemos presumir que la escala utilizada no es la más adecuada para identificar prejuicio entre niños de 8 a 12 años. De acuerdo al componente amenaza y rechazo del exogrupo, que hace referencia a la inferioridad genética por las diferencias étnicas de los miembros del exogrupo; en el caso de los niños de emigrantes, como de los no emigrantes, no hay diferencias étnicas que marquen una posición social fuertemente diferenciada para ser considerados como grupos distintos; es decir, tanto el exogrupo como el endogrupo pertenecen, en este caso, al mismo grupo étnico; por lo que se puede entender que en este aspecto, era improbable obtener resultados que sean reveladores de prejuicio.

En el segundo componente, evitación al contacto íntimo con los miembros del exogrupo, tampoco se encontró indicios de prejuicio, a pesar que en la adaptación de la escala para esta investigación, se precauteló en describir situaciones cotidianas de intimidad propias de los niños, como hacer tareas escolares conjuntamente. Delgado y Contreras (2008) dicen que por las capacidades cognitivas propias del niño, la conducta prosocial aumenta; se incrementa la necesidad de formar parte de un grupo en el cual se comparte conductas, valores y normas. En la investigación se puede entender que las conductas prosociales se facilitan al no existir diferencias étnicas entre los dos grupos. En este sentido, es difícil que los ítems utilizados para estos componentes proporcionen datos que denoten la presencia de prejuicio manifiesto. En el tercer componente, negación de las capacidades y habilidades, los niños que pertenecen al endogrupo no ven obstáculo en la condición de ser niños de emigrantes y que éstos puedan ser buenos estudiantes o asumir responsabilidades dentro del aula; al respecto, el proceso permanente de socialización, así como la necesidad de los niños de pertenecer a un grupo, no da lugar -en estas edades- a minimizar o negar capacidades en sus pares. Los indicadores permiten concluir la ausencia de prejuicio sutil. La escala de estereotipos, en la cual se utiliza un lenguaje más coloquial para los niños, permite identificar de manera clara la presencia de estereotipos que si son percibidos por los niños de emigrantes.

\section{CONCLUSIONES}

Siendo el estereotipo un elemento específico del esquema cognitivo de gran fuerza sobre la información social que reciben los niños como lo dice Fiske (2004), la investigación encontró que tanto los estereotipos positivos como negativos están cargados con atributos negativos que son percibidos de manera evidente por los niños de emigrantes, siendo más notoria la percepción de las niñas con respecto a los varones, especialmente en lo que se refiere a los prejuicios positivos. Esta percepción de los estereotipos en los niños de emigrantes, podría tener algunas explicaciones. Una puede ser el papel del discurso social proveniente del entorno familiar de los niños de no emigrantes; de los medios de comunicación; del propio proceso de socialización que se da en el medio escolar entre otras- los que van conformando un conjunto de creencias que terminan estereotipando a los miembros del exogrupo. El diseño del instrumento posibilitó la mejor comprensión de los ítems y consecuentemente el ubicarse en opciones que detectan el estereotipo. Para verificar lo expresado anteriormente, es necesario avanzar con nuevas investigaciones con adolescentes y adultos, para confirmar la validez de las escalas utilizadas y conocer la evolución del prejuicio en estos grupos etarios. Una característica que se pudo detectar en el proceso de investigación, es que los atributos negativos que son percibidos por los niños de emigrantes, no son reconocidos como prejuicio o como construcciones que les incomode y posiblemente les afecte. Si bien este trabajo no tuvo como objetivo conocer estos aspectos y el impacto social y emocional que produce en los niños; sin embargo, son temas que pueden ser abordados en investigaciones posteriores. 


\section{BIBLIOGRAFÍA}

Allport, G.W., 1962. La naturaleza del prejuicio. Editorial Eudeba, Buenos Aires, Argentina.

Benedictis, G., G. Calfat, 2010. Migration and school attendance: Can remittances compensate for distance to school? San Fernando, Azuay, Ecuador.

Brigham, J.C., 1971. Ethnic stereotypes. Psychological Bulletin, 76, 15-38. En: Montes Berges, B., 2008. Discriminación, prejuicio, estereotipo: conceptos fundamentales, historia de su estudio y el sexismo como nueva forma de prejuicio. Revista Electrónica Iniciación a la Investigación de la Universidad de Jaén, 3, 1-16.

Casal, S., 2006. Los estereotipos y los prejuicios: cambios de actitud en el aula de L2. Estudios de Lingüística Inglesa Aplicada (ELIA), 6, 135-149.

Cronbach, L.J., 1951. Coefficient alpha and the internal structure of tests. Psychometrika, 16(3), $297-$ 334.

Delgado, B., A. Contreras, 2008. Desarrollo social y emocional: desde los seis a los doce años. En: Delgado, B. (Coord.). Psicología del desarrollo: desde la infancia a la vejez. McGraw Hill, Madrid, España.

Fiske, S.T., 2004. Social being: A core motives approach to Social Psychology. John Wiley \& Sons, New York, USA.

García, M., M. Navas, I. Cuadrado, 2003. Los turistas se divierten, los inmigrantes trabajan: Una descripción de dos grupos sociales a través del dibujo infantil. Int. J. Psychol. Psycholog. Ther., 1, 59-79.

Gaviria, E., I. Cuadrado, M. López, 2009. Introducción a la Psicología Social. Sanz y Torres, Madrid, España.

INEC, 2008. Caracterización Sociodemográfica y Económica de las y los Emigrantes Ecuatorianos. Instituto Nacional de Estadísticas y Censos, Quito, Ecuador.

INEC, 2010. VII Censo de Población y VI de Vivienda. Instituto Nacional de Estadísticas y Censos, Quito, Ecuador.

Montes Berges, B., 2008. Discriminación, prejuicio, estereotipo: conceptos fundamentales, historia de su estudio y el sexismo como nueva forma de prejuicio. Revista Electrónica Iniciación a la Investigación de la Universidad de Jaén, 3, 1-16.

Myers, D., 2000. Psicología Social (6ta Ed.). McGraw-Hill, Santa Fé de Bogotá, Colombia.

Ortiz, W., D. Banegas, F. Cabrera, C. López, G. Luzuriaga, C. Roberto, V. Villacrés, 2007. Incidencia del hecho migratorio en los procesos socio-educativos de niños y niñas con padres migrantes de los cantones Girón, Gualaceo y Cuenca. Universidad de Cuenca, Cuenca, Ecuador.

Pettigrew, T.F., R.W. Meertens, 1995. Subtle and blatant prejudice in Western Europe. Eur. J. Soc. Psychol.,25, 57-75.

Rueda, J., M. Navas, 1996. Hacia una evaluación de las nuevas formas del prejuicio racial: las actitudes sutiles del racismo. Revista de Psicología Social, 11, 131-149.

Tajfel, H., J. Turner, 1979. An integrative theory of intergroup conflict. En: Austin, W.G., S. Worchel (Eds.). The social psychology of intergroup relations Brooks/Cole Publ. Co., Monterey, CA, USA, p. 33-46.

Uriel, E., J. Aldás, 2005. Análisis multivariante aplicado. Thomson Editores, Madrid, España. 